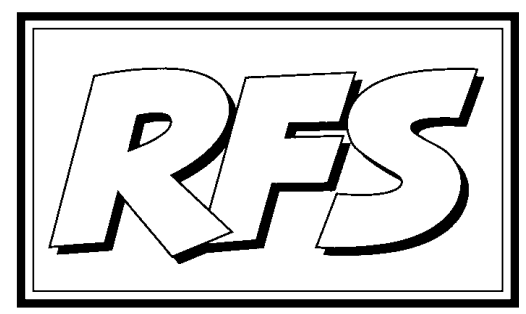

Revista de Fomento Social, 58 (2003), 765-769

\title{
Primera conferencia anual de la red latinoamericana de estudios sobre integración y comercio
}

Pedro CALDENTEY DEL POZO ${ }^{1}$

Los días 6 y 7 de noviembre de 2003 se celebró en Barcelona la primera reunión de la Euro-Latin Study Network on Integration and Trade (Red eurolatinoamericana deestudios so bre integración y comercio) bajo el patrocinio del la Oficina especial del Banco Interamericano de Desarrollo (BID) y el Instituto para la integración Latinoamericana y el Caribe (INTAL) del BID con sede en Buenos Aires. El acto se celebró en la Universidad Pompeu Fabra y fue organizado por el Centro para la Recerca en Economía Internacional (CREI) de la Universidad Pompeu Fabra en colaboración con el Kiel Institute for World Economics (KIEL) y el Robert Schuman Centre for Advanced Studies of the European University Institute (RSCAS).

1 Profesor del Departamento de Economía, Ciencias J urídicas y Sociología de ETEA. Director de la Fundación ETEA para el Desarrollo y la Cooperación. 
La reunión ponía en marcha la primera actividad de esta red promovida por el BID con la intención de reforzar los enlaces del mundo académico latinoamericano con el europeo y de propiciar la definición de puntos de investigación comunes. La conferencia se orientó en torno a los debates sobre la integración y el comercio.

Con un enfoque exclusivamente académico y de contenido notablemente ortodoxo, la reunión tuvo tres sesiones dedicadas a las dimensiones macroeconómicas de la integración regional, a la ampliación de la Unión Europea y las políticas de ajuste durante la transición, y al impacto de la integración regional en el crecimiento y la convergencia económica.

La reunión contó con la presencia de un selecto grupo de miembros de universidades europeas y latinoamericanas y con representantes del BID, CEPAL, Comisión Europea, FMI, y otras instituciones.

Tras la inauguración del acto por Andreu Mas-Colell (Consejero de universidades, investigación y sociedad de la información de la Generalitat de Catalunya), por Carlo Binetti (Representante especial del BID en Europa) y por J uan J osé Taccone (Director del INTAL-BID), la reunión comenzó con una conferencia deAndréSapir, profesor dela Universidad Libre de Bruselas. André Sapir ha alcanzado una cierta notoriedad como miembro del Grupo de asesores de Romano Prodi y coordinador del Ilamado Informe Sapir que propone una reorientación importante de las políticas y el presupuesto de la Unión Europea. La conferencia anunciaba algunas lecciones para América Latina pero se concentró exclusivamente en presentar el contenido del informe.

Sapir expuso su análisis sobre el menor crecimiento de las economías europeas frente al de la economía estadounidense y destacó las dificultades de la UE para impulsar un modelo de crecimiento dinámico. En su opinión, el crecimiento debe ser la primera prioridad de la UE como condición fundamental para sostener el modelo social europeo y garantizar el reto de la ampliación. Éste y el cumplimientos de los compromisos del Consejo Europeo de Lisboa son los principales retos de la UE según Sápir.

El informe recoge una agenda de 6 puntos de la que se derivan 35 recomendaciones. Los 6 puntos son los siguientes:

1. Hacer más dinámico el mercado único.

2. Reforzar la investigación en I+D. 
3. Mejorar el desarrollo de la política macroeconómica.

4. Rediseñar las políticas europeas de convergencia y crecimiento.

5. Mejorar el gobierno económico de la UE.

6. Reorganizar el presupuesto comunitario con criterios de crecimiento y solidaridad.

Su conocido informe sugiere definir ingresos a partir de impuestos de dimensión europea y una reorganización radical del presupuesto de gastos. La actual distribución de gastos (fundamentalmente centrada en la política agraria común-PAC, $45 \%$ del presupuesto total- y en la política de cohesión regional -30\%-) se transformaría para dedicarse a tres políticas: crecimiento, convergencias y reforma. Lo que supondría, entre otras cosas la eliminación de la PAC.

En el coloquio se mencionó la ausencia en el informe, reconocida por el prof. Sapir, de análisis sobre la PAC. En su opinión, todo el mundo reconoce la necesidad de limitar la importancia presupuestaria de la PAC. Las discrepancias reales tendrían más que ver con la necesidad de graduar su progresiva reducción y con el intento de alargarla lo más posible.

La primera sesión sobre las dimensiones macroeconómicas de la integración regional contó con la comunicación de J uan Carlos Berganza, Roberto Chang y Alicia García Herrero, del Banco de España, sobre la "Liability Dollarization, Net Worth Effects, and Contractionary Devaluations". Contó también con la comunicación de Giancarlo Corsetti (Universidad de Roma III y Yale University, USA), Philippe Martin, (Universidad de Paris I y CERAS, Paris, France) y Paolo Pesenti, (Reserva Federal del Banco de New York) sobre la "Globalización y sus mecanismos de transición".

La primera sesión concluyó con dos comunicaciones más. La de Antonio Fatás y llian Mihov (INSEAD, Fontainebleau, Francia) sobre "La Política Fiscal y la Unión Monetaria Europea", que introdujo algunos interesantes comentarios sobre la pertinencia del plan de estabilidad y crecimiento de la UE y otros más críticos sobre su inflexibilidad e inadaptación a las circunstancias de cada país. Y con el trabajo de Dirk Niepelt (IIES, Stockholm University, Suecia) sobre "Los conflictos de la distribución intrageneracional: el papel de los requerimientos de equilibrio fiscal".

La segunda sesión, sobre la ampliación de la Unión Europea y las políticas de ajuste durante la transición, reunió 4 comunicaciones. 
En primer lugar, Carlo Altomonte (Università Bocconi de Milán y Katholieke Universiteit Lovaina, Bélgica) expuso su trabajo sobre la "Integración económica regional y la ubicación de la empresas multinacionales". En segundo lugar, Ansgar Belke (Universidad de Hohenheim, Alemania) presentó su trabajo sobre la "Volatidad de los tipos de cambio y el crecimiento del empleo: evidencia empíricas desde las economías europeas".

En tercer lugar, Helena Marques y Hugh Metcalf (Loughborough University, Reino Unido) expusieron su trabajo "Un modelo de gravedad aplicado al impacto de la migración del trabajo en el comercio sectorial en una UE ampliada". Finalmente, Iulia Traistaru (University of Bonn, Germany), Peter Nijkamp y Simonetta Longhi (Free University of Amsterdam, Holanda) desarrollaron el trabajo "La integración económica, la especialización regional y la concentración de industrias en los países candidatos".

La tercera sesión, sobre el impacto de la integración regional sobre la convergencia y el crecimiento contó con otras cuatro aportaciones. En primer lugar, Gino A. Gancia, (CREl y Universidad Pompeu Fabra) presentó "Globalización, divergencia y Stagnation". En segundo lugar, Hubert Kempf (CNRS y Université Paris I, Panthéon Sorbonne) y Stéphane Rossignol, (Université de Versailles y CNRS, Francia) expusieron el tema "Crecimiento, inequidad e integración: un análisis de política económica".

En tercer lugar, Nicole Madariaga y Sylvie Montout (University of Paris I, Panthéon Sorbonne-M aison des Sciences Economiques, Francia) y Patrice Ollivaud (OCDE) presentaron "Convergencia regional, liberalización comercial y Agglomeration de actividades: un análisis de los casos de NAFTA y MERCUSOR". Finalmente, Gianmarco I. P. Ottaviano (Università di Bologna, Italia) y J acques-François Thies (CERAS, Francia y Université Catholique de Louvain, Bélgica) trataron sobre "Agglomeration and Economic Geography".

Especial interés tuvieron los comentarios a la tercera sesión por parte de Omar Licandro (Robert Schuman Centre for Advanced Studies of the European University Institute -RSCAS- Florencia).

Cerraron las jornadas Robert Devlin (Deputy Manager, Integration and Regional Programs Department, BID) y Antoni Estevadeordal (Principal Trade Economist, BID).

El título de la conferencia resultó ser algo engañoso y más partiendo del BID como organizador principal. La agenda de la reunión estuvo más bien 
volcada en los intereses académicos europeos (la metodología era una comunicación europea y un comentarista latinoamericano) y en un enfoque ortodoxo de la economía aplicada. Sólo los comentarios de Omar Licandro hicieron un esfuerzo de aplicación a los debates de libre comercio e integración en América Latina.

La reunión final sobre la evolución dela red comentó to dos estos aspectos. Esta red planea ampliar el enfoque a otras disciplinas y a la aplicación de políticas. 